\title{
Alpha Spectrometry Applications with Mass Separated Samples
}

\author{
M.P. Dion ${ }^{\mathrm{a}, 1, *}$, Gregory C. Eiden ${ }^{\mathrm{a}}$, Orville T. Farmer III ${ }^{\mathrm{a}}$, Martin Liezers ${ }^{\mathrm{a}}$, \\ John W. Robinson ${ }^{\mathrm{a}}$ \\ ${ }^{a}$ Pacific Northwest National Laboratory Richland, WA 99354
}

\begin{abstract}
${ }^{241} \mathrm{Am}$ has been deposited using a novel technique that employs a commercial inductively coupled plasma mass spectrometer. This work presents results of high-resolution alpha spectrometry on the ${ }^{241} \mathrm{Am}$ samples using a small area passivated implanted planar silicon detector. We have also investigated the massbased separation capability by developing a ${ }^{238} \mathrm{Pu}$ sample, present as a minor constituent in a ${ }^{244} \mathrm{Pu}$ standard, and performed subsequent radiometric counting. With this new sample development method, the ${ }^{241} \mathrm{Am}$ samples achieved the intrinsic energy resolution of the detector used for these measurements. There was no detectable trace of any other isotopes contained in the ${ }^{238} \mathrm{Pu}$ implant demonstrating the mass-based separation (or enhancement) attainable with this technique.
\end{abstract}

Keywords: alpha spectrometry, ICP-MS, actinide, Am-241

\section{Introduction}

Alpha spectrometry is a common analytical technique for the detection of alpha emitting radionuclides. The main reasons for this are low backgrounds, high detection efficiency and relatively low-cost. Other parameters drive the choice of the specific detector. The summary prepared by Vajda et al. (Vajda et al., 1987) provides a well-written overview of alpha spectrometry applications, detector options, etc.

Room temperature silicon detectors with ion implanted contacts ("windows") are the most common choice amongst detectors used in alpha spectrometry due to their thin dead layers (50-100 nm) which minimize energy straggling. Furthermore, they are offered in a variety of sizes, provide good energy resolution, rugged contacts that can be easily cleaned and high detection efficiency. The energy resolution of the detector improves with decreasing the

\footnotetext{
${ }^{*}$ Corresponding author

Email address: michael.dion@pnnl.gov (M.P. Dion)

${ }^{1}$ Address: P.O. Box 999 MSIN: J4-65 Tel.: +1-509-372-6983
} 
active area at the cost of geometrical (detection) efficiency. High-resolution alpha spectrometry involves the production of samples which are thin and free of contaminants and a small-area PIPS detector that attains $<15 \mathrm{keV}$ full-widthat-half-maximum (FWHM) energy resolution.

Pacific Northwest National Laboratory (PNNL) has internally funded a project to investigate the feasibility of using the inherent mass-based separation capabilities of a commercial inductively coupled plasma mass spectrometer (ICP-MS) to reduce some of the tedious chemical separation procedures needed for certain radiometric techniques. This work discusses new results of the Atomic Mass Separation for Enhanced Radiation Detection Measurements (MS-RAD) project. We previously reported (Dion et al., 2014) the results of two ${ }^{241} \mathrm{Am}$ samples ("implants") and the results of energy resolution improvement using low-resolution alpha spectrometry. New results using a high-resolution alpha spectrometer substantiates our previous results and also allows comparison of the two MS-RAD samples. Furthermore, a mass 238 (AMU) implant was derived from a ${ }^{244} \mathrm{Pu}$ NIST standard where ${ }^{238} \mathrm{Pu}$ was a minor constituent. An analysis is performed to determine the level of enhancement of the ${ }^{238} \mathrm{Pu}$ signature of the MS-RAD implant compared to an electrodeposited sample created from the raw NIST standard.

\section{Experimental Methods}

\subsection{ICP-MS Implants}

The details of the implant apparatus used in the quadrupole ICP-MS were previously described in detail (Dion et al., 2014; Liezers et al., 2014). A brief description of the method is supplied here. The ICP-MS was a modified model PQ Excell (VG-Elemental) with an S-option interface where the sample introduction area, ICP and plasma sampling interface were enclosed by a custom fume hood designed specifically for the analysis of radiological samples. The system has a nominal ion transmission efficiency of $0.024 \%$. The standard electron multiplier of the ICP-MS was removed and replaced with a custom standoff. A copper metal block was centered on the standoff and positioned $1 \mathrm{~mm}$ behind the quadrupole exit ion lens. A $15.9 \mathrm{~mm}$ diameter stainless steel planchette was mounted on the copper block with conductive silver epoxy where the ion of interest could be implanted.

Two ${ }^{241} \mathrm{Am}$ MS-RAD samples were developed for the high-resolution alpha spectrometry derived from a commercial gamma standard solution with an activity of $\sim 3.7 \mathrm{E} 4 \mathrm{~Bq} \mathrm{~g}^{-1}$. The implanted samples were targeted to have an activity of $\approx 0.1 \mathrm{~Bq}$. The difference between the two samples was the potential difference applied to the planchette and exit ion lens during implantation. For the "low-bias" sample, the exit ion lens was held at $30 \mathrm{~V}$ and the planchette was held at ground. In this configuration, the implanted ion current could be monitored through a feedthrough via an external electrometer. For the "high-bias" sample, the exit ion lens was held at ground potential and the implant was held at $-1500 \mathrm{~V}$; the ion current could not be monitored since this potential difference was too large for the electrometer. The stock ${ }^{241} \mathrm{Am}$ solution was diluted 
to a concentration of $1.04 \mathrm{ppb}$ in a $10 \mathrm{~mL}$ volume using $0.3 \mathrm{Molar} \mathrm{HCl}$ for the low-bias sample, and the high-bias sample used a diluted solution of $11.6 \mathrm{ppb}$ to reduce the implant time to $18 \mathrm{~min}$. The ICP-MS parameters for the ${ }^{241} \mathrm{Am}$ implants are summarized in Table 1 .

To investigate the mass-based separation capability of the technique, a mass 238 (AMU) implant was created from a dilution of a ${ }^{244} \mathrm{Pu}$ standard (National Bureau Standards Standard Reference Material 996 (NIST, 2014)). The timedecayed isotopic atomic abundance (as of Sept. 2014) of the ${ }^{244} \mathrm{Pu}$ standard is shown in Table 2 (Robinson et al., 2014), half-life values and uncertainties were taken from ENDF (Chadwick et al., 2011). As seen in Table 2, several plutonium isotopes at varying abundances are present, however, mass 238 was selected as the implant species. Room temperature alpha spectroscopy cannot fully deconvolve the alpha particles of ${ }^{239} \mathrm{Pu}$ and ${ }^{240} \mathrm{Pu}$ or ${ }^{238} \mathrm{Pu}$ and ${ }^{241} \mathrm{Am}$. Therefore, if one wants to quantify (without deconvolution) the amount of ${ }^{238} \mathrm{Pu}$ in a sample that contains ${ }^{241} \mathrm{Am}$, a chemical separation would be performed to remove the americium. We selected mass 238 because there was enough activity in $\approx 600 \mathrm{pg}$ of the standard to make a detection after implantation and the influence of the ${ }^{238} \mathrm{Pu}$ to the ${ }^{241} \mathrm{Am}$ energy region would be observable in an unseparated sample (i.e., the activities of ${ }^{238} \mathrm{Pu}$ and ${ }^{241} \mathrm{Am}$ are within an order of magnitude). In contrary, the amount of ${ }^{239} \mathrm{Pu}$ in the standard would have eliminated this opportunity.

The stock concentration of SRM-996 was $3 \mathrm{ppm}$ in $0.5 \mathrm{M} \mathrm{HCl}$ with initial activity and isotopic ratios measured on May 17, 1980. A $10 \mathrm{ml}$ volume of the standard was prepared to a concentration of $50 \mathrm{ppb}$ in $0.4 \mathrm{M}$ HCL. The implant ion current was experimentally determined with $500 \mathrm{ppb}$ solution of $\mathrm{Lu}$. The $\mathrm{Lu}$ solution corresponded to an implant ion current of $26 \mathrm{pA}$ on a Keithley 6517B Electrometer; this equates to an incident ion flux of $3.25 \times 10^{8}{ }^{175} \mathrm{Lu}^{+}$ ions $\mathrm{sec}^{-1} \mathrm{ppm}^{-1}$. The ICP-MS operating parameters are given in Table 1. The ${ }^{244} \mathrm{Pu}$ diluted standard solution was aspirated through the ICP-MS for $5 \mathrm{hrs}$ and $42 \mathrm{~min}$ with the quadrupole set to admit mass 238.05. The solution waste from the spray chamber was recycled back into the starting solution to allow the extended implant time as actual solution consumption rate is only $\approx 0.025 \mathrm{~g} \mathrm{~min}^{-1}$. At the end of the implant period $0.5 \mathrm{ml}$ of the solution remained.

\subsection{Comparative Samples}

A stippled sample was created from the ${ }^{241} \mathrm{Am}$ gamma standard for energy resolution comparison to the MS-RAD samples. The standard was diluted in

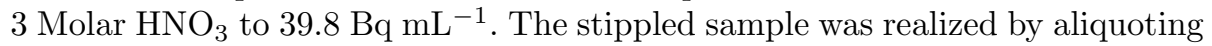
$\approx 5 \mu \mathrm{L}$ of the solution to the center of a $15.9 \mathrm{~mm}$ diameter stainless steel disk and allowed to air-dry. The stipple was targeted to have approximately the same activity of ${ }^{241} \mathrm{Am}$ as the ICP-MS implants, i.e., 0.1 Bq.

For analysis of the mass-based separation capability of the MS-RAD method, an electrodeposited plutonium sample was created from the stock ${ }^{244} \mathrm{Pu}$ standard. A serial dilution of the $3 \mu \mathrm{g} \mathrm{g}^{-1}{ }^{244} \mathrm{Pu}$ stock solution in $3 \mathrm{M} \mathrm{HNO}_{3}$ was prepared, from which an aliquot $(570.5 \mathrm{pg})$ of the dilution was transferred to 
Table 1: A summary of the ICP-MS operating conditions used for the ${ }^{241} \mathrm{Am}$ and ${ }^{238} \mathrm{Pu}$ implants.

\begin{tabular}{llll}
\hline & ${ }^{238} \mathrm{Pu}$ & ${ }^{241} \mathrm{Am}$ & Unit \\
\hline ICP RF Power & 1350 & 1350 & Watts \\
Argon Cool Flow & 12 & 13 & $\mathrm{~L} \mathrm{~min}^{-1}$ \\
Argon Auxiliary Flow & 1.2 & 0.8 & $\mathrm{~L} \mathrm{~min}^{-1}$ \\
Argon Nebulizer Flow & 1.0 & 0.95 & $\mathrm{~L} \mathrm{~min}^{-1}$ \\
Solution Uptake & 0.2 & 0.2 & $\mathrm{~mL} \mathrm{~min}{ }^{-1}$ \\
Spray Chamber Temp. & +2 & +3 & ${ }^{\circ} \mathrm{C}$ \\
Spray Chamber Type & Impact & Impact & \\
& Bead & Bead & \\
Nebulizer & Quartz & Quartz & \\
(AR35-1-FM02E) & Concentric & Concentric & \\
Sample Cone Aperture & 1.0 & 1.0 & $\mathrm{~mm}$ \\
Skimmer Cone Aperture & 0.7 & 0.7 & $\mathrm{~mm}$ \\
Expansion Chamber Press. & 1.9 & 1.9 & $\mathrm{mbar}$ \\
Analyzer Press. & $9.0 \times 10^{-7}$ & $9.0 \times 10^{-7}$ & $\mathrm{mbar}$ \\
Extraction Lens Potential & -550 & -550 & $\mathrm{~V}$ \\
Ion Lens (L1) & +6 & 5 & $\mathrm{~V}$ \\
Ion Lens (L2) & -40 & -40 & $\mathrm{~V}$ \\
Ion Lens (L3) & -200 & -200 & $\mathrm{~V}$ \\
Hexapole Bias & -5 & -3 & $\mathrm{~V}$ \\
Focus & +22 & +28 & $\mathrm{~V}$ \\
Differential Aperture & -100 & -75 & $\mathrm{~V}$ \\
Ion Lens (D1) & -35 & -39 & $\mathrm{~V}$ \\
Ion Lens (D2) & -110 & -168 & $\mathrm{~V}$ \\
Quadrapole Bias & -2.0 & -1.0 & $\mathrm{~V}$ \\
\hline
\end{tabular}


a teflon vial. The aliquot was quantitatively transferred from the vial into a $50 \mathrm{~mL}$ glass beaker using three $1 \mathrm{~mL}$ aliquot rinses of Optima grade $3 \mathrm{M} \mathrm{HNO}_{3}$. Two $\mathrm{mL}$ of $0.36 \mathrm{M} \mathrm{NaHSO}_{4}$ was added to the sample and subsequently dried on a hot plate. Following the drying procedure, $5 \mathrm{~mL}$ of Optima grade concentrated nitric acid was added and then the mixture was wet ashed (i.e., heated on a hot plate and concentrated hydrogen peroxide was added drop-wise) then brought to dryness again. The sample was transferred to the electrodeposition cell using $0.75 \mathrm{M} \mathrm{H}_{2} \mathrm{SO}_{4}$ (initially with $5 \mathrm{~mL}$ then three $2 \mathrm{~mL}$ rinses). Once the sample was $\mathrm{pH}$ adjusted, a platinum wire anode was inserted in the cell about $1 \mathrm{~cm}$ from the disc. The electrodeposition was performed for $1.5 \mathrm{hr}$. Subsequently, the cell was quenched using $1.5 \mathrm{M} \mathrm{NH}_{4} \mathrm{OH}$ then rinsed with $0.1 \mathrm{M} \mathrm{NH}_{4} \mathrm{OH}$ two times and deionized water three times. The cell was disassembled and the disc was rinsed with deionized water and set to air dry.

Table 2: The time-decayed isotopic atomic abundance [\%] and alpha activity of the ${ }^{244} \mathrm{Pu}$ standard used in this work as of Sept. 2014. The table only contains isotopes and/or daughters with an atomic abundance greater than $0.001 \%$. The alpha activity is based on $570 \mathrm{pg}$ of the ${ }^{244} \mathrm{Pu}$ standard.

\begin{tabular}{lll}
\hline Isotope & Atoms [\%] & Alpha Activity $[\mathrm{mBq}]$ \\
\hline${ }^{234} \mathrm{U}$ & $0.00119 \pm 0.002$ & $1.56 \times 10^{-3} \pm 0.31 \times 10^{-3}$ \\
${ }^{236} \mathrm{U}$ & $0.00245 \pm 0.00002$ & $3.35 \times 10^{-5} \pm 0.02 \times 10^{-5}$ \\
${ }^{237} \mathrm{~Np}$ & $0.00255 \pm 0.002$ & $3.78 \times 10^{-4} \pm 0.08 \times 10^{-4}$ \\
${ }^{238} \mathrm{Pu}$ & $0.00381 \pm 0.001$ & $13.8 \pm 2.8$ \\
${ }^{239} \mathrm{Pu}$ & $0.0340 \pm 0.001$ & $0.445 \pm 0.013$ \\
${ }^{240} \mathrm{Pu}$ & $0.675 \pm 0.004$ & $32.4 \pm 0.2$ \\
${ }^{241} \mathrm{Pu}$ & $0.0174 \pm 0.0003$ & - \\
${ }^{241} \mathrm{Am}$ & $0.0721 \pm 0.002$ & $52.2 \pm 1.1$ \\
${ }^{242} \mathrm{Pu}$ & $1.32 \pm 0.004$ & $1.11 \pm 0.01$ \\
${ }^{244} \mathrm{Pu}$ & $97.87 \pm .008$ & $0.374 \pm 0.001$ \\
\hline
\end{tabular}

\subsection{Alpha Spectroscopy}

The measurements of the alpha energy spectrum were performed in a Canberra Alpha Spectrometer-Model 7401. The 7401 was mainly used as a vacuum chamber with vacuum gauge because control over analog shaping time and output pulse shape was needed to obtain ideal energy resolution. Therefore, an Ortec 142A charge-sensitive preamplifier was connected to a right angle adapter which minimized the input cable length from the detector to preamplifier and bypassed the internal electronics of the 7401 . The output of the $142 \mathrm{~A}$ was fed to an Ortec 572A spectroscopy amplifier. The bipolar output of the 572A was analyzed by an Amptek MCA-8000A Pocket Multichannel Analyzer (MCA) and energy spectra were recorded by a laptop PC. The 7401 vacuum chamber was evacuated by a dry scroll pump and achieved ultimate vacuum of a couple mtorr.

The ${ }^{241} \mathrm{Am}$ samples developed using the ICP-MS implant method achieved the intrinsic energy resolution of the large-area silicon detector (20 keV FWHM) 
shown in our previous results (Dion et al., 2014). A high-resolution spectrometer was assembled to further investigate if any differences between the two ICP-MS prepared samples (i.e., applied bias) affected the alpha spectroscopy energy resolution. The detector used for these measurements was a Planar Implanted Planar Silicon (i.e., PIPS ${ }^{\circledR}$ by Canberra) model PD25-10-500AM; $25 \mathrm{~mm}^{2}$ active area, $500 \mu \mathrm{m}$ thick and $10 \mathrm{keV}$ FWHM energy resolution at $5486 \mathrm{keV}$ with $0.5 \mu$ s shaping time. A comparison of the alpha energy spectrum of the high-bias MS-RAD sample is compared to the stipple sample in Figure 1 at a source-to-detector distance (SDD) of $4.7 \mathrm{~mm}$. Compared to the MS-RAD sam-

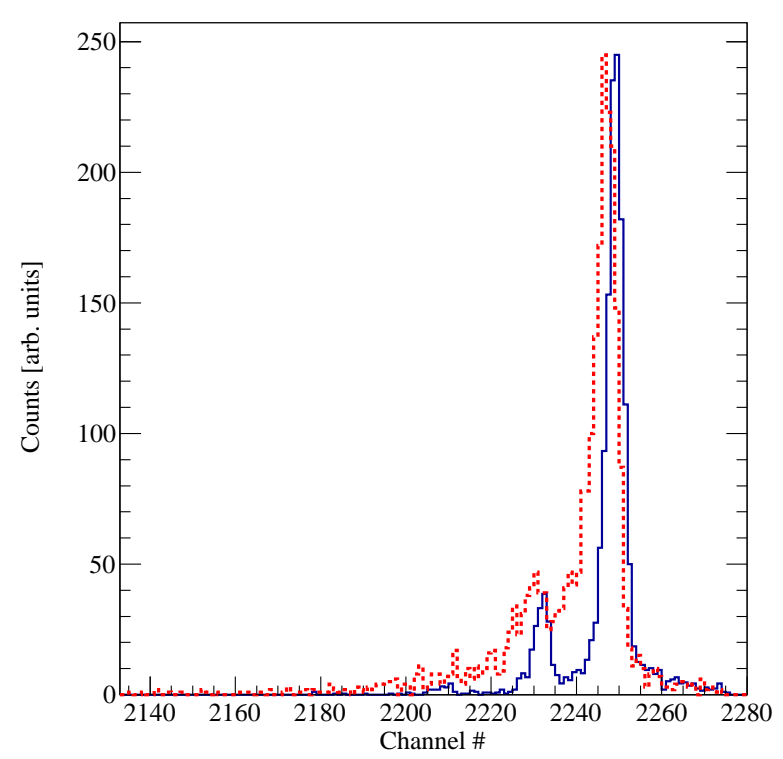

Figure 1: Alpha energy spectra of the high-bias MS-RAD implant (blue solid line) and the stippled ${ }^{241} \mathrm{Am}$ sample (red dotted line). The spectra have been peak height normalized. It is apparent that the energy straggling imparted by the MS-RAD technique is minimal while the stipple sample shows significant broadening of the $5485.6 \mathrm{keV}$ alpha of ${ }^{241} \mathrm{Am}$ most likely due to the carrier matrix of the stipple.

ple, the stipple sample peak has shifted 4 channels to a lower energy and was broadened by $3 \mathrm{keV}$ FWHM. The peak widths of several radiometric measurements are summarized in Table 3. The high-bias MS-RAD sample was counted at SDDs of 3.2, 4.7, 7.2 and $11.2 \mathrm{~mm}$ and the spectra obtained did not exhibit any change in energy resolution suggesting the observed energy resolution is due to the intrinsic resolution of the detector. Within the variance of the measurements, both MS-RAD samples achieve the lower-limit energy resolution of the small area PIPS detector used in these measurements while the stippled sample exhibited broadened line width. There is a slight gain $(6 \%)$ in energy resolution measured in the low-bias sample in comparison to the high-bias sample that is 
statistically significant. This could be an implication that the ${ }^{241} \mathrm{Am}$ ions in the low-bias sample are not "buried" into the planchette as much as the high-bias sample.

We investigated the ion implant depth and the effect of peak width broadening by Monte-Carlo modeling. The experimental geometry was defined and included the detector dead layer, SDD, silicon active area, vacuum pressure and vacuum chamber dimensions. The simulations were performed with Geant4

Table 3: Statistical averages and standard deviation of the FWHM energy resolution of the low- and high-bias MS-RAD samples and the stippled sample of ${ }^{241} \mathrm{Am}$.

\begin{tabular}{ll}
\hline Sample & FWHM $[\mathrm{keV}]$ \\
\hline low-bias & $9.69 \pm 0.12$ \\
high-bias & $10.31 \pm 0.06$ \\
stipple & $14.17 \pm 0.04$ \\
\hline
\end{tabular}

(Agostinelli et al., 2003) and the ${ }^{241}$ Am decay was handled using the Radioactive Decay Module. The source term was $1 \times 10^{6}$ decays of ${ }^{241} \mathrm{Am}$ of an isotropic point source aligned about the center axis of the detector (i.e., silicon). The total energy deposited per event in the silicon was stored in a ROOT (Brun and Rademakers, 1997) histogram. This histogram was Gaussian smeared to return the same FWHM energy resolution as measured with the low-bias sample. The ion implant depth from the high-bias sample was approximated by trial and error simulations by varying the thickness of a disc of stainless steel located $0.02 \mathrm{~mm}$ above the source term. It was found that a thickness $75 \AA$ of stainless steel above the isotropic point source reproduced the experimentally observed difference in energy resolution between the two MS-RAD samples.

The implant depth is an area of concern for MS-RAD. The radiometric counting of a particular isotope could be heavily influenced by the attenuation/straggling imposed by the implant method and would require further efficiency corrections. Biasing the implant was examined as a way to "steer" ions to a multi-implant target which would allow multiple implants from one input solution. Further studies have to be performed that quantify the depth of the implanted ion as a function of implant bias.

Creating alpha spectroscopy samples using a modified ICP-MS produced a clean, thin matrix unattainable with the traditional sample preparation methods compared in this work. However, it should be noted that other techniques have been developed that can attain thin sample matrices and FWHM energy resolution equal to what is reported in this work Becerril-Vilchis et al. (1996); Tsoupko-Sitnikov et al. (2000). MS-RAD could prove a valuable tool as an alternative separation procedure for isotopes of plutonium that have alpha energy overlaps or separating isotopes that have similar chemical behavior. 


\subsection{Mass-Based Separation of ${ }^{238} \mathrm{Pu}$}

A PIPS PD150-12-500AM (150 $\mathrm{mm}^{2}$ active area, $12 \mathrm{keV}$ FWHM energy resolution at $5486 \mathrm{keV}$ and $500 \mu \mathrm{m}$ thick) was used for the alpha spectroscopy measurements to compare the MS-RAD implant to the electrodeposited (ED) sample created from the ${ }^{244} \mathrm{Pu}$ standard. The larger detector was used for increased detection efficiency. Both samples were counted with a source-to-detector distance of $4.7 \mathrm{~mm}$ and to obtain counting statistics uncertainty $<5 \%$; the mass implant was counted for 12 days and the ED sample for 5 days.

The background subtracted spectra are shown in Figure 2. The data is

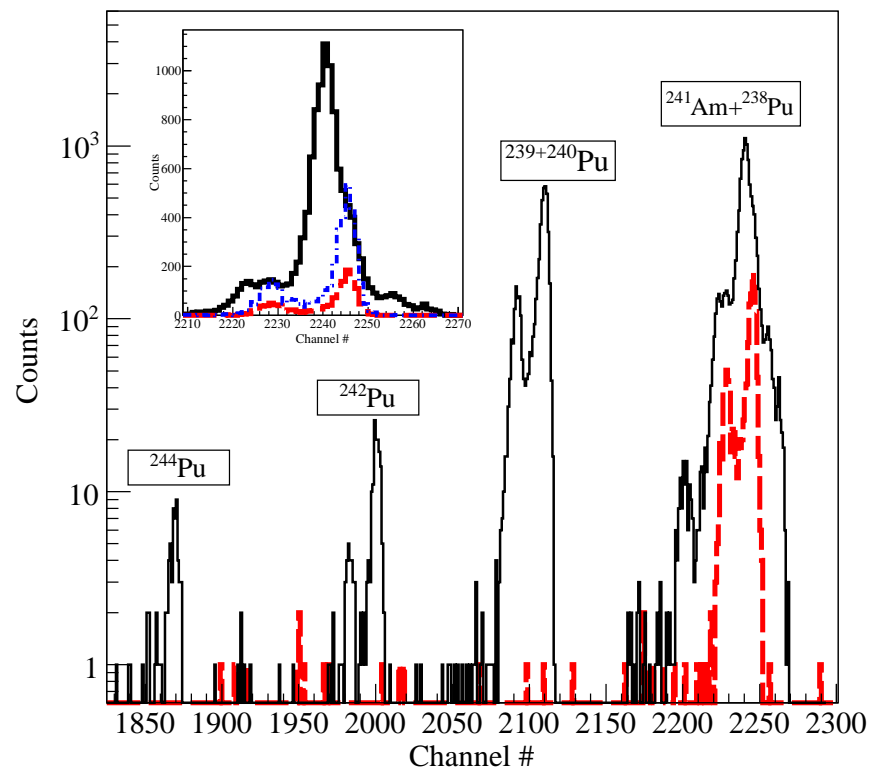

Figure 2: Alpha energy spectra of the MS-RAD implant (dotted red curve) and the ED sample (black curve). Due to the unresolvable separation of ${ }^{238} \mathrm{Pu}$ and ${ }^{241} \mathrm{Am}$ with room temperature silicon detectors, the ${ }^{238} \mathrm{Pu}$ alpha particle energy is convoluted in the ${ }^{241} \mathrm{Am}$ peak of the ED sample. See text for description of plot inlay. Background has been subtracted from both energy spectra.

shown as uncalibrated to eliminate any ambiguity that may occur due to the variances in sample construction that could lead to energy straggling, however, alpha particle energy has been confirmed with a certified three isotope alpha calibration source. It is clear from comparison of the two energy spectra that all other isotopes of plutonium $(239,240,242$ and 244) have been removed from the MS-RAD implant. This would suggest that mass 241 has also been removed (because of the mass window imposed by the plutonium species) and the only contribution to the energy spectrum could be mass $238\left({ }^{238} \mathrm{Pu}\right)$. Having prior knowledge of ICP-MS performance, the most likely mass to tail into the 
${ }^{238} \mathrm{Pu}$ implant would be mass 239 . From Table 2 , the activity ratio of ${ }^{238} \mathrm{Pu}$ to ${ }^{239} \mathrm{Pu}$ is $31: 1$. Therefore, if ${ }^{239} \mathrm{Pu}$ had tailed into the mass 238 implant, $\approx 46$ counts would be expected in the energy region of channel 2050-2120 in Figure 2. However, two counts are present in this energy region which indicates $>20$ times removal of mass 239 to 238 .

The inset in Figure 2 is a linear y-axis plot focused on channels 2210-2270 of the ED and MS-RAD spectra. This is the alpha particle region of ${ }^{241} \mathrm{Am}$ and ${ }^{238} \mathrm{Pu}$. The ED sample (solid black curve) in this channel region has a dominant alpha particle peak interfered by a higher energy alpha particle. The dashed red curve is the MS-RAD mass 238 implant. To qualitatively confirm that the high-side asymmetric shape of the main alpha peak of the ED sample in this region of the inset is attributable to ${ }^{238} \mathrm{Pu}$ in the ${ }^{244} \mathrm{Pu}$ standard, the MSRAD spectrum has been scaled based on the ratio of peak height of the lower energy alpha contribution of ${ }^{238} \mathrm{Pu}$ (channel 2226) in the ED sample to the peak height in the MS-RAD implant (blue dash-dotted curve) and clearly the scaled MS-RAD implant overlaps this high-side asymmetry very well. Furthermore, the higher energy peaks beyond channel 2250 in the ED sample are alpha-conversion electron coincidences and higher energy low-yield alpha particles from the decay of ${ }^{241} \mathrm{Am}$. Again, the omission of these peaks from the MS-RAD implant further confirms the removal of mass 241 (this is more visible in the log y-axis plot).

The level of signal enhancement (or separation) can be quantified if the activity of ${ }^{238} \mathrm{Pu}$ in the ED sample can be deconvoluted from the ${ }^{241} \mathrm{Am}$ alpha distribution. This avoids the dependency on the SRM-996 data sheet and also corrects for any discrepancies during the electrodeposit sample preparation. The energy spectrum of the ED sample was analyzed using the Gold (Morháč and Matoušek, 2011; Gold, 1964) deconvolution algorithm implemented via ROOT's TSpectrum class. The deconvolution function takes a detector "response" and "source" spectrum and calculates the resulting deconvoluted spectrum. The response of the silicon detector was modeled as a convolution of a Gaussian with one exponential (Bortels and Collaers, 1987) as:

$$
f(x)=\frac{A}{2 \cdot \tau} e^{\left(\frac{x-\mu}{\tau}+\frac{\sigma^{2}}{2 \tau^{2}}\right)} \operatorname{erfc}\left(\frac{1}{\sqrt{2}}\left(\frac{x-\mu}{\sigma}+\frac{\sigma}{\tau}\right)\right) .
$$

The shape parameters are the width of the Gaussian $\sigma$, and $\tau$ to describe the exponential low-energy peak tailing, $A$ is the peak area (number of counts) and $\mu$ is the peak position. The deconvolution algorithm returned the center location of each peak which was used as an initial guess. The constant "amplitude" term $A$ was adjusted for each peak to roughly describe the energy spectrum, $\sigma$ and $\tau$ were set to $2.00(\mathrm{chn})$ and 3.08 , respectively.

There are seven peaks of interest in the alpha spectrum between channels 1990-2270: two main alpha peaks for each isotope $(5499.0$ and $5456.3 \mathrm{keV}$ $(99.89 \%)$ for ${ }^{238} \mathrm{Pu}$ and 5485.6 and $5442.8 \mathrm{keV}(97.9 \%)$ for $\left.{ }^{241} \mathrm{Am}\right),{ }^{241} \mathrm{Am}$ has two peaks at higher energy that are low intensity alpha particles and coincident events (Siiskonen and Pöllänen, 2006) and a low-energy alpha particle at 5388 $\mathrm{keV}(1.66 \%)$. A function to describe the seven peaks was defined based on 
Equation 1 (i.e. $f(x)_{1}+f(x)_{2}+\ldots$ ). The shape parameters of the function were fixed as previously described and the peak centers were initially set to the result of the Gold algorithm but were allowed to change. The function was fit to the energy spectrum and resulted in the dash-dotted (magenta) curve in Figure 3. The parameters from the final fit were back substituted into individual functions and are plotted in Figure 3 to show the contribution of each isotope; ${ }^{241} \mathrm{Am}$ peaks are solid red and ${ }^{238} \mathrm{Pu}$ peaks are dashed green.

The uncertainties stated in this analysis are the standard deviation uncertainties of the fitted peaks returned from MINUIT James (2014) implemented in ROOT. The ratio of activity based on the described fit analysis of ${ }^{238} \mathrm{Pu}$ and ${ }^{241} \mathrm{Am}$ determined by the area term in Equation 1 is $0.27 \pm 0.04$ and $0.73 \pm$ 0.03 , respectively compared to $0.209 \pm 0.043$ and $0.791 \pm 0.036$ derived from Table 2. We find reasonable agreement when considering the assumptions given above and error propagation between the relative alpha activity found by deconvolution and peak fitting and the reported time-decayed values of the standard. Therefore, using the values found for total counts from the convoluted ${ }^{238} \mathrm{Pu}$

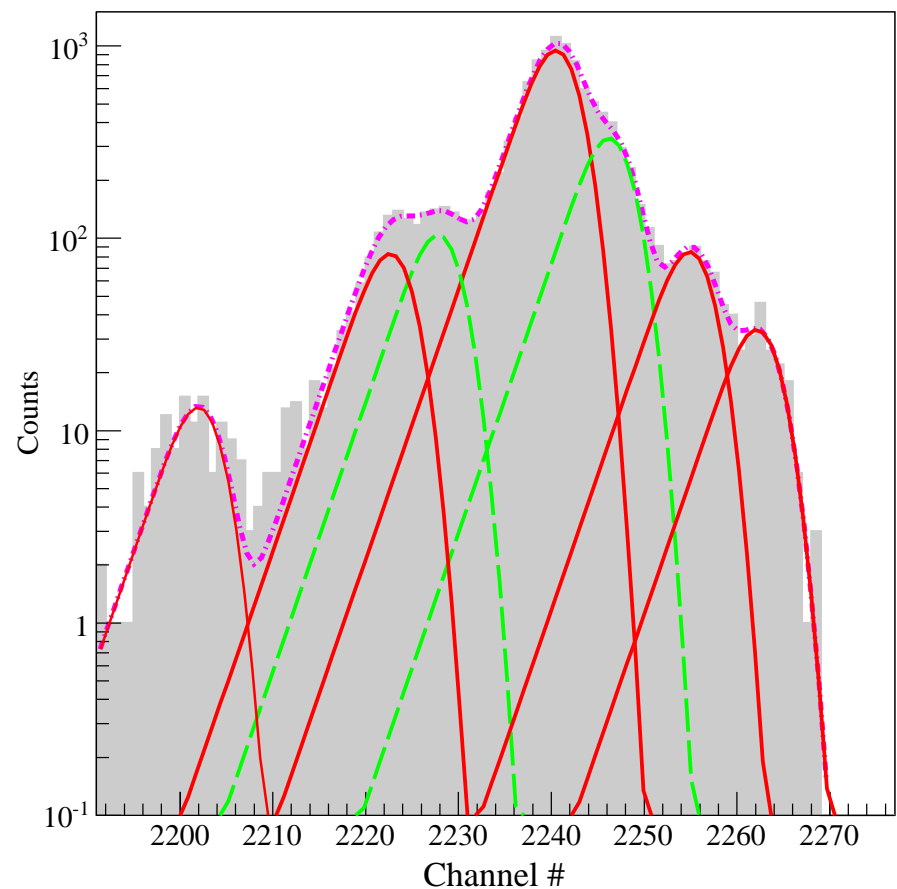

Figure 3: The experimental alpha spectrum of the ED sample (gray filled histogram). The individual contributions from ${ }^{241} \mathrm{Am}$ (solid red curves) and ${ }^{238} \mathrm{Pu}$ (dashed green curve) are plotted along with the sum function (dash-dotted magenta curve).

259 and ${ }^{241} \mathrm{Am}$ alpha region from the ED sample, we can estimate the level of mass 
238 enhancement (or removal) achieved on the ${ }^{244} \mathrm{Pu}$ standard used in this work. Based on count times, the estimated activity from the ${ }^{238} \mathrm{Pu}$ implant and the ED sample are given in Table 4. Therefore, the implant procedure used in this work has enhanced the ${ }^{238} \mathrm{Pu}$ signal from the ${ }^{244} \mathrm{Pu}$ isotopic standard by $159 \pm$ 27 based on alpha activity.

Table 4: The estimated activity of the ED and ${ }^{238} \mathrm{Pu}$ implant developed in this work based on alpha spectroscopy and the peak fitting in Figure 3. These are used to estimate the level of separation achieved in this work. "The "other" contribution for the ${ }^{238} \mathrm{Pu}$ implant is the integral of the energy spectrum excluding the contribution of ${ }^{238} \mathrm{Pu}$; no other peaks are visible in the spectrum.

\begin{tabular}{ll}
\hline${ }^{244} \mathrm{Pu}$ ED: & \\
\hline Isotope & Count Rate $\left[\mathrm{s}^{-1}\right]$ \\
\hline${ }^{241} \mathrm{Am}$ & $20.2 \pm 0.3$ \\
${ }^{238} \mathrm{Pu}$ & $7.6 \pm 0.3$ \\
${ }^{239,240,242,244} \mathrm{Pu}$ & $13.8 \pm 0.2$ \\
\hline${ }^{238} \mathrm{Pu}$ Implant: & \\
\hline Isotope & Count Rate $\left[\mathrm{s}^{-1}\right]$ \\
\hline${ }^{238} \mathrm{Pu}$ & $1.33 \pm 0.04$ \\
Other* & $0.037 \pm 0.006$ \\
\hline
\end{tabular}

\section{Conclusions}

A high-resolution alpha spectrometer was assembled to compare two MSRAD ${ }^{241} \mathrm{Am}$ implants that were developed with different accelerating potentials and to compare to a conventional stipple source. There was a slight improvement in energy resolution achieved with the low-bias implant compared to the highbias while a $32 \%$ and $27 \%$ improvement was found between the stipple and low- and high-bias implants, respectively. The difference in energy resolution between the MS-RAD implants could be due to the influence of the implant bias on the actual depth that the ions are implanted into the planchette. This feature requires a more thorough investigation but preliminary simulations indicate a $75 \AA$ implant depth reproduces the energy resolution difference between the two implants. Each of the MS-RAD ICP-MS implanted ${ }^{241}$ Am samples achieved the intrinsic energy resolution of the detector used in this work even at the closest SDD and no improvement was found by increasing the SDD. This achievement is likely due to the elimination of any carrier matrix in the sample development. The efficiency of the ICP-MS $(0.02 \%)$ limits the applications that can be targeted by this method but still allows for the preliminary quantitative steps necessary for this technique.

The mass-based separation and subsequent implant capability using a modified ICP-MS for radioisotopes was tested with a NIST SRM-996 ${ }^{244} \mathrm{Pu}$ standard. 
This test has shown the removal of all other plutonium (and americium) isotopes from a mass 238 (AMU) implant. This ${ }^{238} \mathrm{Pu}$ implant was analyzed via alpha spectroscopy and compared to an ED sample created from the unseparated standard. An unfolding fit technique was applied to the convoluted energy region of ${ }^{241} \mathrm{Am}$ and ${ }^{238} \mathrm{Pu}$ of the ED sample and it was shown that the MSRAD method was able to enhance the ${ }^{238} \mathrm{Pu}$ signature of the ${ }^{244} \mathrm{Pu}$ standard by $159 \pm 27$. Therefore, MS-RAD has the ability to either eliminate or reduce the amount of chemical separation necessary in samples that contain radioisotopes of the same (or different) elements which exhibit radiometric overlap.

Future work needs to develop a method of quantification starting from the front-end injected sample to radiometric counting. Reducing analytical timelines by the elimination of chemical separations requires improved efficiency of the implant technique. The efficiency $(0.02 \%)$ of the current instrument would only be applicable to original samples with high sample activity since most of the isotope of interest would not be implanted. This low efficiency reduces the activity of the implant, which increases the count time needed to reach statistical significance, and therefore lengthens the analysis timeline. PNNL is pursuing the purchase of a high-resolution multi-collector ICP-MS (with radioactive isotope capability) which will enable consecutive implants and an increase (10-50 times) in efficiency.

\section{Acknowledgements}

We wish to thank Janet Cloutier (PNNL) for her work as project manager, Dawn Lucas and May-Lin Thomas (PNNL) for developing the electrodeposited samples, Shannon Morley (PNNL) for developing the stipple samples and Charles Barinaga (PNNL) for useful discussions and insight. The research described in this paper was conducted under the Ultra-Sensitive Nuclear Measurements Initiative, a Laboratory Directed Research and Development Program at Pacific Northwest National Laboratory, a multi-program national laboratory operated by Battelle for the U.S. Department of Energy.

\section{References}

N. Vajda, P. Martin, K. Chang-Kyu, Alpha Spectrometry, in: D. Pines (Ed.), Handbook of Radioactivity Analysis, Addison-Wesley Publishing, New York, NY, 1987, pp. 79-185.

M. P. Dion, M. Liezers, O. T. Farmer III, B. W. Miller, S. Morley, C. Barinaga, G. Eiden, Improving alpha spectrometry energy resolution by ion implantation with ICP-MS, J. Radioanal Nucl Chem 303 (2014) 877-884.

M. Liezers, O. T. Farmer III, M. P. Dion, M. L. Thomas, G. C. Eiden, The production of ultra-high purity single isotopes or tailored isotope mixtures by icp-ms, International Journal of Mass Spectrometry (2014).

NIST, 2014. http://www.nist.gov/srm/. 
J. W. Robinson, M. P. Dion, G. C. Eiden, O. T. Farmer, M. Liezers, RadICalc: A program for estimating radiation intensity of radionuclide mixtures, J. Radioanal Nucl Chem (2014) 1-6.

M. Chadwick, et al., ENDF/B-VII.1 nuclear data for science and technology: Cross sections, covariances, fission product yields and decay data, Nuclear Data Sheets 112 (2011) 2887 - 2996. Special Issue on ENDF/B-VII.1 Library.

S. Agostinelli, et al., Geant4a simulation toolkit, Nuclear Instruments and Methods in Physics Research Section A: Accelerators, Spectrometers, Detectors and Associated Equipment 506 (2003) 250 - 303.

R. Brun, F. Rademakers, ROOT - an object oriented data analysis framework, Proceedings AIHENP'96 Workshop, Lausanne, Sep. 1996, Nucl. Instr. and Meth. A 389 (1997) 81-86. See also http://root.cern.ch/.

A. Becerril-Vilchis, A. Cortés, F. Dayras, J. de Sanoit, A method for the preparation of very thin and uniform -radioactive sources, NIM A 369 (1996) 613 -616 .

V. Tsoupko-Sitnikov, F. Dayras, J. de Sanoit, D. Filossofov, Application of rotating disk electrode technique for the preparation of $\mathrm{np}$, pu and am sources, Applied Radiation and Isotopes 52 (2000) 357 - 364 .

M. Morháč, V. Matoušek, High-resolution boosted deconvolution of spectroscopic data, J. Comput. Appl. Math. 235 (2011) 1629-1640.

R. Gold, AN ITERATIVE UNFOLDING METHOD FOR RESPONSE MATRICES, Argonne National Lab, 1964. URL: http://www.osti.gov/scitech/ servlets/purl/4634295, doi:10.2172/4634295.

G. Bortels, P. Collaers, Analytical function for fitting peaks in alpha-particle spectra from Si detectors, Appl. Radiat. Isot. 38 (1987) 831-837.

T. Siiskonen, R. Pöllänen, Alpha-electron and alpha-photon coincidences in high-resolution alpha spectrometry, Nuclear Instruments and Methods in Physics Research Section A: Accelerators, Spectrometers, Detectors and Associated Equipment 558 (2006) 437 - 440.

F. James, MINIUT, 2014. http://lcgapp.cern.ch/project/cls/ work-packages/mathlibs/minuit/index.html. 